\title{
Military Based Automated Guided Vehicle System
}

\author{
Dharmesh Visaroliya, Helly Patel, Bhavya Dhoru, Karan Dewani, Ajay Patel
}

\begin{abstract}
The interest of this research is to provide a solution to the use of Unmanned Ground Vehicles in military applications. This paper model such an Automated Guided Vehicle (AGV) that can not only be used as load carriers but also as transportation and surveillance vehicles with an ability to communicate, too. From border surveillance to carrying arms \& ammunitions for the camps, there are a lot many applications of this AGV. Furthermore, changing the intelligence of the system to fully automated possible applications are explored in this paper. With this intelligence system, vehicles can provide constant updates about its surroundings, and the data shared with peer vehicles will include information on potential and sudden obstacles on the route. Communication of an Unmanned Aerial Vehicle or any other AGV can be achieved. The study concludes that the objective of an autonomous vehicle system in military and other applications can be achieved by this model.
\end{abstract}

Keyword: Automated guided vehicle, military vehicles, sensor fusion, swarm technology,unmanned grounded vehicle.

\section{INTRODUCTION}

Automated systems have been used in the military for decades. Advanced machinery and robotic systems have proved to be a great aid for the beings living at the borders, with their lives at stake, all the time. There has been a huge development in military vehicles as well. Many unmanned ariel and ground vehicles have taken over the difficult tasks of soldiers. Innovation in "military robotics" started with Nikola Tesla (1856-1943) [1]. In the early days, UAVs were used to carry heavy payloads of 60 to $100 \mathrm{~kg}$ of high explosives for certain demolition activities [2]. Then drones came into the picture for airstrikes with their attacking mechanisms.

The iRobot launched 510 PackBot in 2002, a tactical mobile robot designed to perform chemical, biological, radiological and nuclear (CBRN) detection, explosive ordnance disposal (EOD), improvised explosive device (IED) detection, checkpoint, and vehicle and personnel inspections apart from surveillance and reconnaissance [3]. This technology is extending further as, robots like Atlas, Avatar, CRACUNS, etc. are designed to replace the army completely [4].

Revised Manuscript Received on May 20, 2020.

Dharmesh Visharoliya, Mechatronics Engineering, G H Patel College of Engineering \& Technology, Anand, India. .Email: dharmeshvishroliya@gmail.com

Helly Patel, Mechatronics Engineering, G H Patel College of Engineering \& Technology, Anand, India. .Email: hellypate1390@gmail.com

Bhavya Dhoru, Mechatronics Engineering, G H Patel College of Engineering \& Technology, Anand, India. Email: dhorubhavya@gmail.com

Karan Dewani, Mechatronics Engineering, G H Patel College of Engineering \& Technology, Anand, India. Email: karandewani98@gmail.com

Ajay Patel, Mechatronics Engineering, G H Patel College of Engineering \& Technology, Anand, India. Email: ajaypatel@gcet.ac.in

Ground forces are to be completely imitated by autonomous vehicles in a few years. Although the researchers have made great contributions in this area, intercommunication between two vehicles is still an area of research. The benefits of AGVs in the military are already enormous but can be further extended if some on board intelligence is implemented on them.

\section{LITERATURE REVIEW}

$\mathrm{UGVs}$ in the military is seen as a great milestone to be achieved in the future. Unmanned vehicles are in use in many applications in the military such as transportation of logistics and deploy vehicles to carry out dangerous missions. But the army needs an AGV with technology smarter than humans, that can continue on its path despite bad weather or tall abducted trees, or unexpected half hanging branches, etc. [5]. This need has led to the research of path planning and localization of AGV dated back in 2018 published by Naveed Ur Rehman, Kundan Kumar, and Ghulam e Mustafa Abro[6]. They have successfully implemented path planning and localization using SLAM technology in AGVs. Another research with approved simultaneous path planning and localization algorithm are done by Demim Fethi and his team [7], by carrying out certain simulations, experiments and also prototyping all into robot Pioneer P3-AT. All these researches were inefficient and inaccurate at some points, and so interfacing of the SLAM Kalman filter and GSM module is suggested in this paper.

The noteworthy researches on object detection have been made by, Xia Hua and his team [8], where they have combined object detection technology with visual salience and human psychology. Their experimental results while compared with the exiting algorithms show that the algorithm proposed by them has more advantages in precision, effectiveness, and simplicity, which with significantly reducing the effectiveness of battlefield camouflage and deception, also achieves rapid and accurate detection of military objects.

But making a vehicle Autonomous will not satisfy all the needs. To compensate for the UGV's disadvantages like low view, a collaboration of UGVs and UAVs is also suggested in this paper. Thus this research paper studies the means of implementing such intelligence in autonomous vehicles, making them tremendously useful in not only transportation but tasks like surveillance, rescuing, path planning, etc. and that can be achieved by making UAVs and UGVs communicate with each other. 


\section{PERIPHERALS TO BE USED}

A. Transparent Robot Smart Car Chassis:

Table I: Specifications of car chassis

\begin{tabular}{|c|c|}
\hline Parameters & Specifications \\
\hline Length $(\mathrm{mm})$ & 245 \\
\hline Width $(\mathrm{mm})$ & 157 \\
\hline Height $(\mathrm{mm})$ & 57 \\
\hline Weight $(\mathrm{kg})$ & 0.35 \\
\hline
\end{tabular}

\section{B. Ultrasonic Sensor [9] and IR Sensor:}

IR sensor helps AGV to sense its surroundings and gathers data if any obstacle is detected in the range of the sensor $(100 \mathrm{~cm}-500 \mathrm{~cm})$ and sends the data directly to the microcontroller [10].

Table II: Specifications of Ultrasonic Sensor

\begin{tabular}{|c|c|}
\hline Parameters & Specifications \\
\hline Operating Voltage(VDC) & 5 \\
\hline $\begin{array}{c}\text { Average Current } \\
\text { Consumption(mA) }\end{array}$ & 2 \\
\hline Frequency(Hz) & 40000 \\
\hline Sensing Angle(Degree) & 15 \\
\hline Max Sensing Distance $(\mathrm{cm})$ & 450 \\
\hline Weight $(\mathrm{gm})$ & 9 \\
\hline
\end{tabular}

\section{Raspberry Pi 3B+ and Pi Camera:}

The model used here is the third generation/iteration of the raspberry pi. It is an ARM-based low-cost and a powerful credit-card-sized single-board computer. Third generation Raspberry $\mathrm{Pi}$ model is used as it provides 10x faster performance compared to the first generation. In addition to this, it also features LAN and Bluetooth connection, making it perfect for UAV communication.

The Raspberry Pi-camera is a standard-issue camera that will be capturing high-definition video at 1920*1080 resolution to provide enough convenience for typical usage on the UAV. The camera uses its own dedicated CSI Interface, designed especially for camera interfacing.

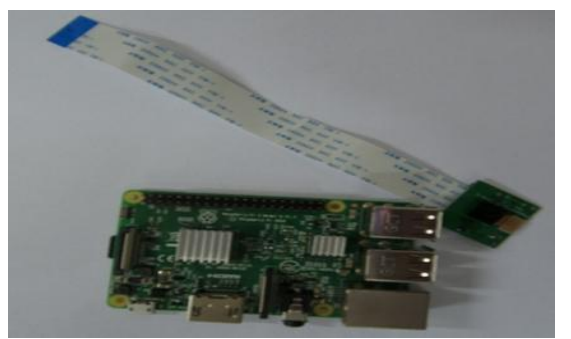

Figure 1: Raspberry Pi 3+ model with Pi camera

\section{Li-Po Battery as a power source:}

Lithium Batteries are preferred here as, with higher discharge rate and high weight vs stored energy ratio, they can be utilized for the task substantially. The issue with its charging is crucial and cannot be ignored.

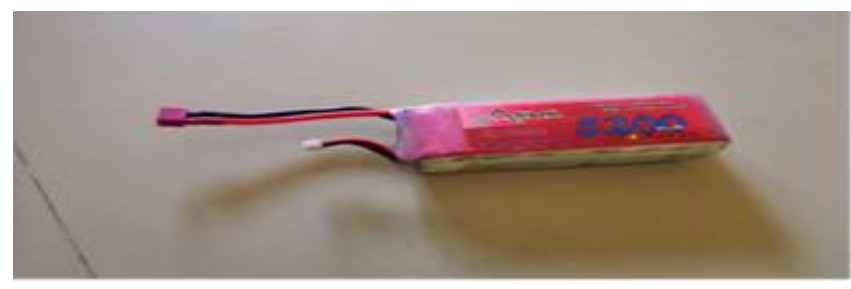

Figure 2: Li-Po Battery (5200 mAh)

E. Motor Driver(L298N) [11]:

Table III: Motor Driver Specifications:

\begin{tabular}{|c|c|}
\hline Parameters & Specifications \\
\hline $\begin{array}{c}\text { Operating } \\
\text { Voltage(VDC) }\end{array}$ & $5 \sim 35$ \\
\hline $\begin{array}{c}\text { Peak } \\
\text { Current(A) }\end{array}$ & 2 \\
\hline $\begin{array}{c}\text { Continuous } \\
\text { Current(mA) }\end{array}$ & $0-36$ \\
\hline $\begin{array}{c}\text { No. of } \\
\text { Channels }\end{array}$ & 2 \\
\hline
\end{tabular}

\section{F. Inertial Measurement Unit (IMU):}

IMU unit is basically a combination of Accelerometer, Gyroscope and on some occasions Magnetometer. It will be used to get data on Body's specific force, angular rate and body's orientation at specific intervals.

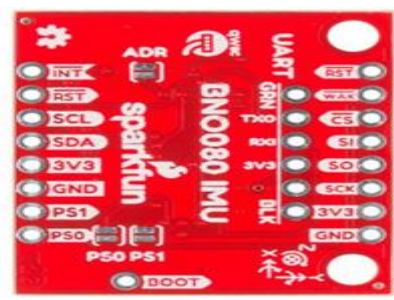

Figure 3: IMU unit

\section{G. UAV:}

It consists of Naza M Lite Flight Controller, 5000 mAh LiPo Battery, F450 Carbon Fiber Frame, Raspberry Pi 3B+ \& Pi Cam, and custom-designed gripper mechanism which helps UAV to sustain an average flight time of $12 \mathrm{~min}$, and 200-250 gm of payload (depending upon wind conditions). The purpose of UAV is to collaborate with ground AGV units and to provide AGVs with the surveillance data which UAV has collected, to detect the enemy location precisely. UAV is also used to carry explosives and can also collect small items from hazardous areas rapidly [12].

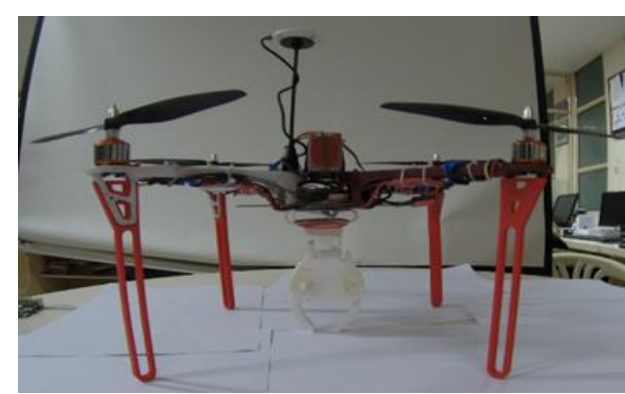

Fig 4-UAV installed with Two-Jaw Custom Gripper 


\section{METHODOLOGY}

A prototyping model is developed to study the results with the above mentioned peripherals and the features and characteristics to be implemented in this model are as follows:

\section{A. Area Surveillance:}

From camped areas to border surveillance, AGVs can be used to make sure, there is no threat in the surroundings. First of all, the area that is to be surveyed, is fed to the UAV. UAV will survey the given area and check if the enemy is present, by differentiating from living and nonliving objects with the help of machine vision which will be concluded by Raspberry Pi Cam mounted on UAV. If an enemy troop is present, it will send the data to Master AGV.

\section{B. Task Allocation:}

With the help of sensors, it will collect the data of the surroundings and send it to the main server, where the data will be processed and accordingly, tasks will be allocated to AGV.

\section{Path Planning and Obstacle Detection:}

No predefined path will be allotted to the vehicle. Using Kalman Filter interfacing with sensors like LIDAR, RIDAR, or a normal camera, it will make its own best-suited route and navigate through the obstacles from an initial point to the given final destination. An intelligent obstacle avoidance system can be used to avoid infinite loop pitfalls of certain hurdles during AGV's movement using a convolutional neural network [13]. Furthermore, a warning system can also be installed for humans in case of emergencies. For this kind of system, various sensors like an ultraviolet sensor, IR sensor, cameras, and proximity sensor can be used for fulling the need of input data to certain algorithms installed to the on-board controller (Raspberry Pi 3B+).

As soon as the AGV is assigned a task, and initial \& final point are resolved, the shortest path can be planned by either of these algorithms:

- Dijkstra algorithm (for slower and simplest paths comparatively) [14].

- $\mathrm{A}^{*}$ algorithm (more effective for smaller areas) [15].

- $\quad \mathrm{D} *$ Lite algorithm (extension to $\mathrm{A}^{*}$ algorithm but more accurate for larger areas) [16].

D*Lite algorithm for larger areas is preferred in military applications.

\section{Data Allocation to Master AGV:}

If action is to be taken, the master AGV gives a command to the slave AGV to move in a specific direction. By using this Master-Slave concept, it will help all the on-board microprocessors to reduce processing time.

\section{E. Image Classifier Algorithm:}

For this, we have used the concept Convolutional Neural Network (CNN) with the help of Python. The program uses Keras library for training the basic $\mathrm{CNN}$ model. By doing

so, the accuracy of the basic programming model increases. The program is then uploaded to Raspberry Pi via the use of the Virtual Network Computing (VNC) server. CNN consists of various key elements, which helps the image to get reduced in size without any loss in its quality whereas, the flattening of the image is done later on. AGVs will be interconnected with each other with the use of Arduino UNO [17] in addition to the SIM7600 GSM module. The precise position of $\mathrm{AGVs}$ and UAVs is done using a GPS module. While using a camera sensor for path planning and object detection, the image classifier algorithm will be used.

\section{F. UGV and AGV Collaboration:}

Communication between UAV and UGV is done here with the help of the ROCI (Remote Object Control Interface) operating system [18]. A high-level OS for programming and managing networks of UAVs and UGVs, and sensors. Each robot is a node that contains many processing and sensing modules and shares different types of services and data to other nodes, i.e., other robots. Complex tasks can be accomplished by connections of several inputs and outputs of specific modules defined in XML.

\section{RESULTS}

For object detection and path planning, the D* Lite algorithm and Kalman filters were tested. While implementing for the UAV and UGV collaboration, the problems of accuracy and reliability were the main concern, so more than one system were to be integrated. Data fusion with decentralized manner [19], ROCI and decentralized active sensor networks [20].

\section{CONCLUSION}

The research carried out, gives the following conclusions:

- Communication between AGVs and UAVs can be proven to be effective by this method for military applications.

- Smarter and a proven system of performing military tasks, like surveillance, defense, and transportation, without causing any fatality, can be developed using master and slave communication concepts.

\section{REFERENCES}

1. P. W. Singer, "Drones don't die - A history of military robots", Available: https://www.historynet.com/drones-dont-die-a-history-ofmilitary-robotics.htm

2. Christopher McFadden, $6^{\text {th }}$ Nov, 2018; “ A brief history on military vehicles including autonomous vehicles”, Available: https://interestingengineering.com/a-brief-history-ofmilitary-robots-including-autonomous-systems

3. "iRobot 510 PackBot Multi-Mission Robot", Available: https://www.army-technology.com/projects/irobot-510-packbotmulti-mission-robot/

4. "10 military robots of the future" Available: https://www.designnews.com/content/10-military-robots-future

5. Sydney J. FreedbergJr.on August 20, 2018 "Army Wants 70 Self-Driving Supply Trucks By 2020", Available: https://breakingdefense.com/2018/08/army-wants-70-self-drivingsupply-trucks-by-2020/ 


\section{Military Based Automated Guided Vehicle System}

6. Naveed Ur Rehman ; Kundan Kumar; Ghulam e Mustafa Abro, 19 April 2018, "Implementation of an autonomous path planning \& obstacle avoidance UGV using SLAM", Available: DOI: 10.1109/ICEET1.2018.8338628

7. Demim Fethi, AbdelkrimNemra, KahinaLouadj and Mustapha Hamerlain, "Simultaneous localization, mapping, and path planning for unmanned vehicle using optimal control", Available: https://doi.org/10.1177/1687814017736653

8. Xia Hua, Xinqing Wang, Dong Wang, Jie Huang and Xiaodong Hu, "Military Object Real-Time Detection Technology Combined with Visual Salience and Psychology", Available: https://doi.org/10.3390/electronics7100216

9. Alessio Carullo and Marco Parvis, An ultrasonic sensor for distance measurement in automotive applications, Politecnico di Torino, Available: doi: 10.1109/JSEN.2001.936931

10. Seong-Soo Kim, Christina Young, Boris Mizaikoff, Miniaturized mid infrared sensor technologies, Ulm -University, Available: doi: 10.1007/s00216- 007-1673-5

11. Tolga Özer, Sinan Kivrak, Yüksel Oğuz, "H Brıdge DC Motor Drıver Design and Implementatıon with using dsPIC30f4011", Available: https://www.researchgate.net/publication/317225711_H_Bridge_DC Motor_Driver_Design_and_Implementation_with_Using_dsPIC30f40 11

12. Luis Ramos, Alejandro Diaz, Daniel Reyes, "Unmanned Ariel Vehicle", Florida International University, Available: https://mme.fiu.edu/wp-content/uploads/2013/04/T7_TBD.pdf

13. Seongkyun Han,JisangYoo, and Soonchul Kwon, "Real-Time Vehicle-Detection Method in Bird-View Unmanned-Aerial-Vehicle Imagery", https://www.ncbi.nlm.nih.gov/pmc/articles/PMC6767679/

14. Xie Yang and Cheng Wushan, AGV path planning based on smoothing A* algorithm, Shanghai University of Engineering (2016), Available: doi: 10.5121/ijsea.2015.6501

15. Guo Qing, Zhang Zheng, Xu Yue, Path planning of Automated Guided Vehicle based on improved Dijkstra algorithm, Beijing University of Chemical Technology (2017), Available: https://www.semanticscholar.org/paper/Path-planning-of-automatedguided-vehicle-based-on-QingZheng/f67aa43cc778836b8d7390f4399be7896c9bd661/figure/3

16. Dae Hwan Kim, Nguyen Trong Hai, Sang Kwun Jeong, A guide to selecting path planning algorithm for automated guided vehicle Pukyong National University, Available: doi: 10.1007/978-3-31969814-4_56

17. Menyhart Jozsef, "Concept of an UGV with Arduino device", Available:

https://www.researchgate.net/publication/273449017_CONCEPT_OF AN UGV WITH ARDUINO DEVICE

18. Dr. Daisy Tang, "Collaboration Between Unmanned Aerial and Ground Vehicles" file:///C:/Users/Helly\%20Patel/Downloads/UAV-UGV.pdf

19. S.GrimeH.F.Durrant-Whyte, "Data fusion in decentralized sensor networks", Available: https://doi.org/10.1016/0967-0661(94)90349-2

20. Alexei Makarenko, Alex Brooks, Stefan Williams, Hugh DurrantWhyte, "A Decentralized Architecture for Active Sensor Networks", ARC Centre of Excellence in Autonomous Systems (CAS), Available:https://www.researchgate.net/publication/4077169_A_dece ntralized_architecture_for_Active_Sensor_Networks

\section{AUTHORS PROFILE}

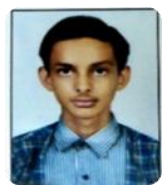

Dharmesh Visharoliya, Mechatronics engineer, member of ISTE student branch at $\mathrm{G} \mathrm{H}$ Patel College of Engineering \& Technology.

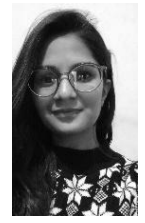

Helly Patel, Mechatronics Engineer, been a member of ISTE student branch at $\mathrm{G} \mathrm{H}$ Patel College of Engineering \& Technology, volunteered and organized TEDxGCET for two years, done IOT projects and applications in product and automotive design area. Worked as fund raiser in Kalam NGO.

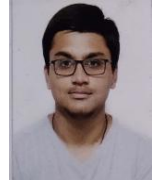

Bhavya Dhoru, Mechatronics Engineer, Member of ISTE student branch at $\mathrm{G} \mathrm{H}$ Patel College of Engineering \& Technology and Kalrav NGO. Achieved best project title for a technical project in college technical festival.

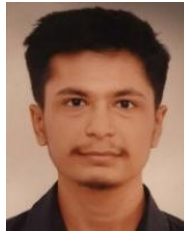

Karan Dewani, Mechatronics Engineer, been a member of ISTE and Prarambh club at $\mathrm{G} \mathrm{H}$ Patel College of Engineering \& Technology and Kalrav NGO. Been a state topper for two years, achieved best project title in college technical festival.

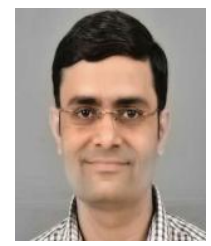

Ajay Patel, Associate Professor in mechatronics department, at G H Patel College of Engineering \& Technology, associated in the research of, robotics, finite element method, automation, Nano-mechanics, etc. Credited in more than 35 papers in reputed journals. 